\title{
Towards A Hybrid Relational and XML Benchmark for Loosely-Coupled Distributed Data Sources
}

\author{
Mahesh B. Chaudhari, Suzanne W. Dietrich, Jennifer Ortiz, Spencer \\ Pearson \\ School of Mathematical and Natural Sciences, Arizona State University
}

\begin{abstract}
There are known benchmarks for the performance evaluation of relational and XML databases. However, there is an increasing demand for database applications that require access to heterogeneous loosely-coupled distributed data sources. This paper presents a hybrid benchmark based on TPC-H where the data sources are heterogeneous. Specifically, the paper describes the design of the relational and XML data sources as well as the query redesign in the LINQ query language, which supports queries over heterogeneous data sources. The results of a performance evaluation of the hybrid benchmark over various database products is included for untyped and typed XML with and without clearing the database cache.
\end{abstract}

Keywords:

benchmark, distributed, heterogeneous, relational, XML, LINQ, TPC-H

\section{Introduction}

Benchmarks have been widely used to evaluate relational databases and applications driven by relational databases $[11,8]$. These benchmarks assess the database implementation within a given product and test environment with respect to the amount of time required to execute the queries and produce the correct results based on various scaling factors of the underlying database instance. There are also benchmarks for assessing the performance of XML databases. However, the performance assessment of systems accessing hybrid data sources, such as relational and XML data, has not been well explored. 
Recent database applications are requiring combined access to different types of distributed data sources to provide answers to complex queries. The term dataspaces $[13,10,9]$ refers to such systems that use a data co-existence approach to handle various data sources, leaving the sources in their original format, and thus, representing a loosely-coupled federation of distributed, heterogeneous sources. A potential example of such a system is a criminal justice scenario that accesses information across jurisdictions involving myriad databases and Web data, such as traffic cameras and alert systems. In this scenario, the data may be stored in relational databases and streams of XML data, formatted according to the National Information Exchange Model (NIEM) [19], which is the broader successor of the Global Justice XML Data Model. The goal of the proposed benchmark is to create a similar data scenario of hybrid, distributed sources using the well-known enterprise of the TPC-H benchmark [24]. Since the 22 queries of the original TPC-H benchmark are written in SQL to query the data stored in relations only, the query language used to exercise these complex queries over the heterogeneous data must be considered. Although SQL/XML represents one possibility to query relational and XML sources stored in a relational database, the objective of the benchmark is to query heterogeneous data sources maintained in their original format. Therefore, the Language INtegrated Query (LINQ) $[16,14,2]$ language was chosen as a declarative query language that supports query specifications over heterogeneous data sources. LINQ is integrated into object-oriented programming languages supported by the .NET Framework, which is available for different operating systems including UNIX-based systems with the Mono library implementation of the framework [25]. LINQ can query heterogeneous data sources because it provides an abstraction layer, parsing the language into expression trees. Using data providers, the query expression tree forms the basis of a translation of the query to the target of the data source. There are data providers for various database products (SQL Server, Oracle, mySQL), Web services such as Amazon [14] and big data [15]. Therefore, the design and generation of the heterogeneous data sources along with the redesigned queries in LINQ form the basis of the proposed hybrid benchmark.

Specifically, this paper describes the design of a hybrid TPC-H benchmark that supports queries over heterogeneous data sources. Section 2 briefly discusses related work. Section 3 provides a brief overview of the TPC$\mathrm{H}$ enterprise and LINQ, by illustrating LINQ using the TPC-H enterprise. Section 4 describes the design and data generation of the heterogeneous data 
sources. The framework for the redesign of the 22 benchmark queries in LINQ is given in Section 5. Section 6 provides a performance evaluation of the benchmark on two anonymous database products with untyped and typed XML as well as cache clearing options. The paper concludes with a discussion.

\section{Related Work}

The Transaction Processing Performance Council (TPC) has been a leading organization developing different types of benchmarks for various database scenarios. TPC has several benchmarks targeting relational databases. For example, TPC-E [23] is an online transaction processing benchmark, emphasizing a database-centric workload based on a financial market with transactions involving brokerage trades and customer interactions. TPC-H [24] is an ad-hoc, decision support benchmark that consists of a suite of businessoriented complex ad-hoc queries and concurrent data modifications for a customer-order database.

There are also XML benchmarks to assess the performance of XML databases. XMach-1 [1] was developed for testing web applications accessing XML data, primarily XML documents containing structured, schema-less data. The benchmark includes a collection of XML queries and updates using the XQuery language. XMach-1 evaluates both native XML database systems and XML-enabled relational DBMS. XMark [22] is another benchmark developed for XML databases and includes an XML Schema specification of the database. The benchmark is based on auctions held for different items in various regions and the people involved in bidding for these auctions. Transaction Processing over XML (TPoX) [20, 26] has been developed to evaluate transaction processing over XML databases, and is based on an online brokerage scenario focusing on XML document processing using XQuery, SQL/XML, XML indexing, XML schema support and XML updates using the XQuery update language specification.

Although the performance assessment of systems accessing loosely-coupled distributed data sources has not been well explored, there is one related research project, Ferry, which has evaluated LINQ queries that access both relational and XML data sources together [12]. The Ferry project combines the relational tables from the TPC-H enterprise and the XML schema from the XMark benchmark into one hybrid enterprise. However, this hybrid enterprise consists of only one XML document with one relationship between 
the relational and XML data, which limits the number of queries that can be benchmarked over the heterogeneous sources. In addition, the goal of the work in [12] was a query compilation technique resulting in an efficient, alternative LINQ query provider. The goal of the work presented in this paper is to leverage the well-known enterprise of the TPC-H benchmark to create a hybrid benchmark with related heterogeneous data sources that are evaluated with respect to the comprehensive decision support queries of the original TPC-H benchmark, redesigned for the hybrid environment.

\section{Overview of the TPC-H Benchmark and LINQ}

The TPC-H benchmark [24] is specifically designed for benchmarking adhoc, decision support systems. A UML conceptual class diagram for the TPC-H enterprise is shown in Fig. 1. Customers place orders for various parts. The line items on the order indicate the part and supplier of the part. Customers and suppliers reside in a nation, which is associated with a given region.

The TPC-H benchmark specification includes a data generation specification as well as a suite of $22 \mathrm{SQL}$ queries. The TPC provides a $\mathrm{C}++$ program to generate the benchmark relational data according to its specification, starting at a $1 \mathrm{~GB}$ database that is scalable to $30 \mathrm{~GB}$. The $22 \mathrm{SQL}$ queries use a rich set of relational operators and represent varying degrees of complexity. These queries extensively use aggregation functions and grouping along with sorting and nested queries to rigorously test the application under consideration. Therefore, the remainder of this section provides a quick overview of aggregation and grouping in LINQ since these features are used later in the characterization of the benchmark queries.

LINQ is a query language that supports querying over heterogeneous data sources. LINQ can query over tuples in a relation, objects in a collection, or elements in XML. LINQ is reminiscent of SQL in that LINQ has select, from, and where clauses. However, the order of these clauses in LINQ reflects the execution order of the query: from, where, and select. The from clause iterates over a collection filtering the query results using the where clause and returning a collection of results structured as specified by the select clause. The from-where-select syntax is translated to a functional syntax underneath, and stored as an expression tree. LINQ providers translate the expression into queries over the corresponding data source when the query 


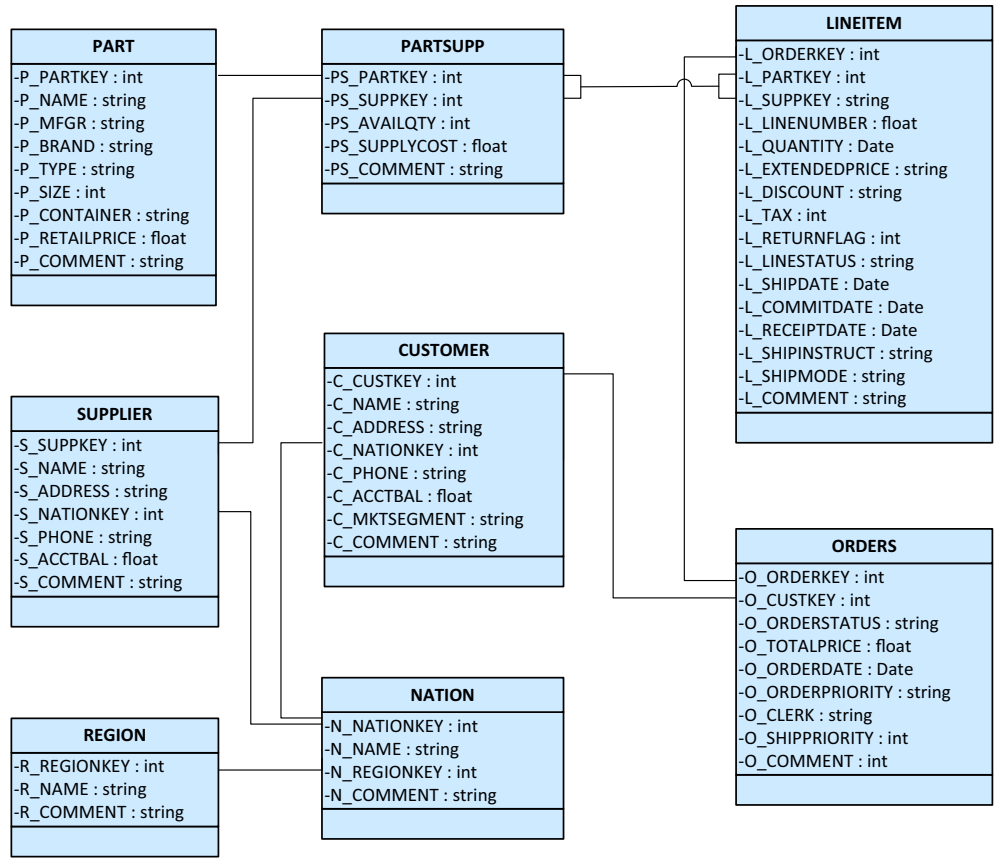

Figure 1: TPC-H UML Diagram. 
is materialized. Using the TPC-H enterprise as shown in Fig. 1, the use of LINQ to query relations and XML will be discussed.

\subsection{Querying Relations}

LINQ is a query language that is integrated into a programming language, so it is case sensitive. Since the TPC-H scripts create the relational database using uppercase table and attribute names, the query examples will follow that case structure. Also, LINQ does not limit a query to a single occurrence of a from or where clause, and these clauses can be intertwined. LINQ also provides let and orderby clauses. A let clause defines a variable value associated with the current iteration, which is similar to a let in XQuery although LINQ requires a query to start with a from clause. The orderby clause is one word in LINQ and can specify any property or list of properties within the scope of the query, meaning that the query can be ordered by properties not specified in the select clause.

Consider the following sample query to find the maximum total of customer orders. This query illustrates a let clause, a nested query, and three occurrences of aggregation. The aggregate functions in LINQ (Count, Average, Max, Min, Sum) are implemented using extension methods, which are methods that have been extended to be applied to more than one class.

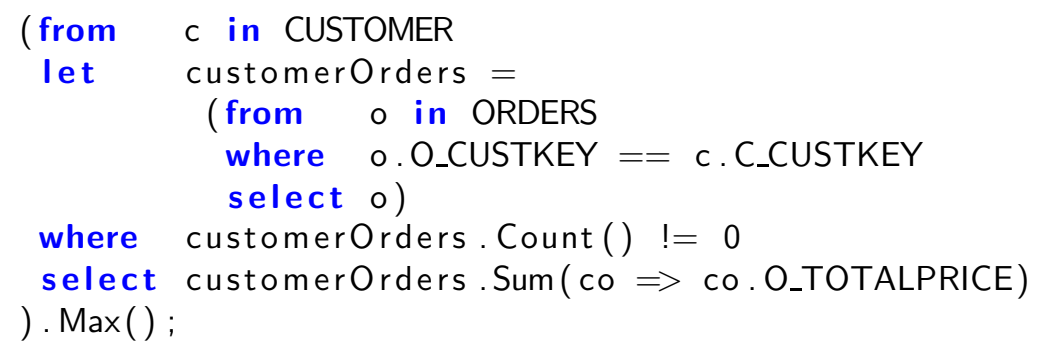

Since the benchmark instance requires that one-third of the customers do not have any orders, the Count extension method is applied to the collection of orders for the customer in the where clause, verifying that the customer has orders to total. The Sum extension method must be applied to a numeric operand and thus, uses the lambda "goes to" syntax, indicated by $=>$, with the variable co ranging over the customerOrders collection to retrieve the attribute O_TOTALPRICE for use in the Sum. The Max extension method then returns the maximum total over the collection of sums.

There is also a grouping capability in LINQ, which has a syntax that is different than SQL. The group clause specifies what to group and what to 
group by and typically includes an into specification that provides a name for iterating over the resulting groups. Consider the following sample query that finds the number of line items on each order along with the information on those line items.

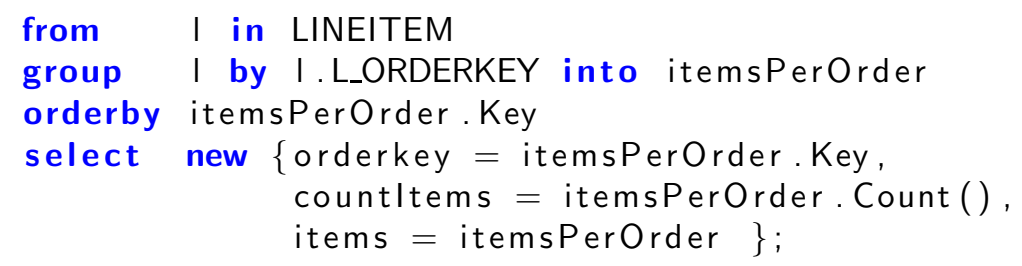

In this sample query, the variable iterating over the tuples in the LINEITEM table is grouped by the L_ORDERKEY attribute value, and the name itemsPerOrder refers to the resulting groups. The results are ordered by the Key associated with the group by, which is I.L_ORDERKEY in this example. This select clause illustrates the creation of a new anonymous type that has three named fields: the value of the order key, the count of the number of line items for the order, and the collection of line items associated with that order. In general, the grouped and grouped by fields may be simple existing types or a new anonymous type that represents a composite field.

\subsection{Querying $X M L$}

The default LINQ provider for XML is for untyped XML, which does not have an associated XML Schema Definition (XSD). Querying untyped XML requires the use of specific methods to navigate the XML Document Object Model with LINQ. The XElement type provides a Load method for loading the XML document into main memory as well as Descendants and Elements methods that return a collection of descendants and elements, respectively, of the current node. Given a parameter, these methods return a collection restricted to the name given. There are also Element and Attribute methods that return an XElement or XAttribute, respectively, based on the parameter name.

Consider the last sample query that finds the number of line items on each order along with the information on those line items. Assume that the data is now an XML data source with an element-based representation of the relational data, where each tuple of a table is enclosed in an element with the table name and nested within are the elements that correspond to the attributes of the table. The data source is loaded using the following 
statement, which also illustrates the implicit typing of the variable $\mathrm{db}$ as XElement:

var $\mathrm{db}=$ XElement. Load (@"TPCH.xml");

The collection of elements that corresponds to each table can be defined as shown using the LINEITEM as an example:

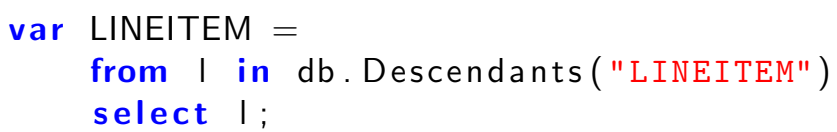

The sample query over XML is very similar to the query over the relational data source. The only syntactic difference is the group by using the value of the L_ORDERKEY element, where the Value member returns the content of the element:

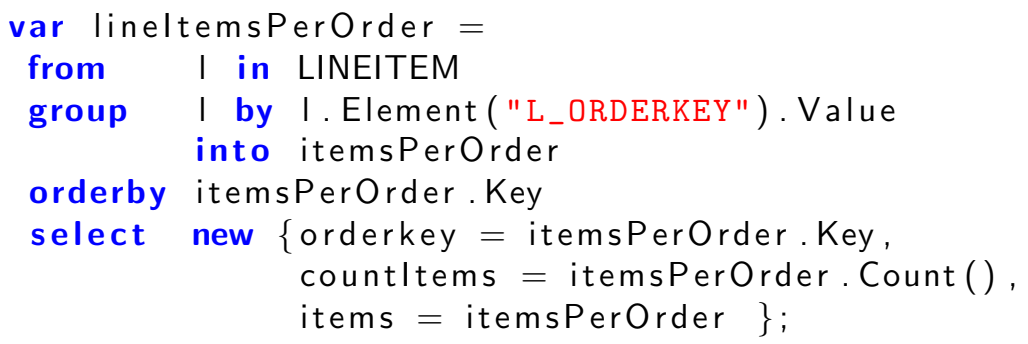

LINQ also supports querying typed XML, meaning that there is an XML Schema Definition (XSD) associated with the XML context. In that case, the explicit navigation methods are unnecessary. Therefore, for the above sample query, the relational version using only dot notation is the same as the typed XML version.

\subsection{Querying relational and XML data sources}

LINQ supports a query specification that allows for joins between relational and XML data sources. The current LINQ default data providers allow for multiple XML data sources but only one relational data source having multiple tables as shown in Fig. 2. Sample heterogeneous queries in LINQ will be provided in the benchmark description, after the design of the heterogeneous data sources for TPC-H is discussed in the next section.

\section{Data Sources for Hybrid Benchmark}

An analysis of the TPC-H enterprise and queries led to the design of the heterogeneous data sources described in this section, which also includes a discussion of the generation of the data sources. 


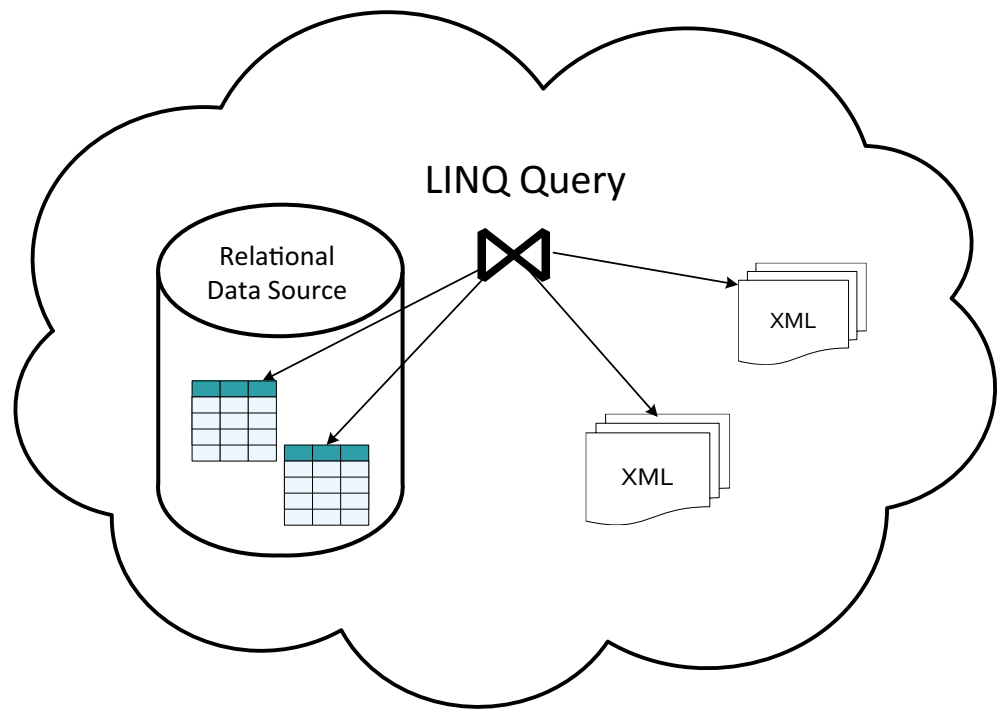

Figure 2: LINQ querying relational and XML data sources.

\subsection{Data analysis and design}

The TPC-H scenario was analyzed to determine whether there was a business use case for deciding which data to store in relational versus XML format. Since the customer and order information is typically entered through the Web, this information became the candidates for storing in XML. The remainder of the data, such as part and supplier, would be maintained in a relational database. With this division of data, the matrix shown in Fig. 3 illustrates which information is accessed in each of the 22 queries in the benchmark. The right-most columns of the figure provide a summary of the query distribution with the proposed heterogeneous data sources, indicating which of the queries are XML only (8), relational only (3), or a hybrid query (11) over both XML and relational data sources.

Based on this business use case and the resulting distribution of queries, further analysis was required to design the proposed CUSTOMER, ORDERS, and LINEITEM XML data sources. The benchmark specification requires that one-third of the customers do not have any orders. Also, orders have exactly one customer and one to seven line items chosen at random. Based on this analysis and an objective of having multiple XML data sources, the customer information is stored in one XML document and the order information along with its associated line items is stored in another XML document. The 


\begin{tabular}{|c|c|c|c|c|c|c|c|c|}
\hline \multirow[b]{3}{*}{ Query } & \multicolumn{8}{|c|}{ TPC-H QUERIES } \\
\hline & \multicolumn{3}{|c|}{ XML } & \multicolumn{5}{|c|}{ RELATIONAL } \\
\hline & CUSTOMER & ORDERS & LINEITEM & PART & PARTSUPP & SUPPLIER & NATION & REGION \\
\hline Q1 & & & $x$ & & & & & \\
\hline Q2 & & & & $x$ & $x$ & $x$ & $x$ & $x$ \\
\hline Q3 & $x$ & $x$ & $\mathrm{x}$ & & & & & \\
\hline Q4 & & $x$ & & & & & & \\
\hline Q5 & $x$ & $x$ & $x$ & & & $x$ & $x$ & $x$ \\
\hline Q6 & & & $x$ & & & & & \\
\hline Q7 & $x$ & $x$ & $x$ & & & $x$ & $x$ & \\
\hline Q8 & $x$ & $x$ & $x$ & $x$ & & $x$ & $x$ & $x$ \\
\hline Q9 & & $x$ & $x$ & $x$ & $x$ & $x$ & $x$ & \\
\hline Q10 & $x$ & $x$ & $x$ & & & & $x$ & \\
\hline Q11 & & & & . & $x$ & $x$ & $x$ & \\
\hline Q12 & & $x$ & $x$ & 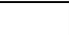 & & & & \\
\hline Q13 & $x$ & $x$ & & & & & & \\
\hline Q14 & & & $x$ & $x$ & & & & \\
\hline Q15 & & & $x$ & & & $x$ & & \\
\hline Q16 & & & & $x$ & $x$ & & & \\
\hline Q17 & & & $x$ & $x$ & & & & \\
\hline Q18 & $x$ & $x$ & $x$ & & & & & \\
\hline Q19 & & & $x$ & $x$ & & & & \\
\hline Q20 & & & $x$ & $x$ & $x$ & $x$ & $x$ & \\
\hline Q21 & & $x$ & $x$ & & & $x$ & $x$ & \\
\hline Q22 & $x$ & $x$ & & & & & & \\
\hline Totals & 8 & 12 & 16 & 8 & 5 & 9 & 9 & 3 \\
\hline
\end{tabular}

\section{SUMMARY}

QUERY DISTRIBUTION

\begin{tabular}{|c|l|l|}
\hline XML & RELATIONAL & HYBRID \\
\hline$X$ &
\end{tabular}

\begin{tabular}{|c|c|c|}
\hline$x$ & & \\
\hline & $\mathbf{X}$ & \\
\hline $\mathbf{X}$ & & \\
\hline$X$ & & \\
\hline & & $\mathbf{x}$ \\
\hline $\mathbf{X}$ & & \\
\hline & & $\mathbf{X}$ \\
\hline & & $\mathbf{X}$ \\
\hline & & $\mathbf{X}$ \\
\hline & & $x$ \\
\hline & $\mathbf{X}$ & \\
\hline$X$ & & \\
\hline $\mathbf{X}$ & & \\
\hline & & $\mathbf{X}$ \\
\hline & & $x$ \\
\hline & $\mathbf{X}$ & \\
\hline & & $\mathbf{X}$ \\
\hline$X$ & & \\
\hline & & $x$ \\
\hline & & $\mathbf{x}$ \\
\hline & & $\mathbf{X}$ \\
\hline $\mathbf{X}$ & & \\
\hline 8 & 3 & 11 \\
\hline
\end{tabular}

Figure 3: Hybrid TPC-H relational and XML data sources. 
relational data sources (PART, PARTSUPP, SUPPLIER, NATION, REGION) remain the same as in the original TPC-H benchmark (as shown in Fig. 1).

\subsection{Data generation}

The data generation $\mathrm{C}++$ program provided by the TPC, known as DBGEN, creates relational data starting at a minimum scale factor of $1 \mathrm{~GB}$ that adheres to the various requirements of the benchmark. An initial functional evaluation was performed on the 1GB version of the benchmark. Unfortunately, there were many queries that did not complete in a reasonable amount of time. Therefore, the decision was made to start the investigation with a smaller scale version of the TPC-H data instance. The DB-GEN program required modifications to generate data for scale factors less than $1 \mathrm{~GB}$ in order to preserve the rules and constraints of the TPC-H benchmark. The size of the database generated for performance evaluation in Section 6 corresponds to a $200 \mathrm{MB}$ version of the relational TPC-H database. In addition, a $1 \mathrm{MB}$ version of the relational database was created for use by students in database courses to explore the relational TPC-H benchmark [21]. Fig. 4 indicates the format of the revised data sources with an abstraction of the referential associations between the sources. Rather than change the $\mathrm{C}++$ program to generate both relational and XML data, LINQ queries transform the identified relational data sources into XML. For customers, orderkeys were nested within a new ORDERS element. Similarly, for orders, the line items associated with an order were nested within a LINEITEMS element. Fig. 5 shows a snippet of the LINQ queries used to transform the relational data into XML, applying a functional construction approach by creating a new XElement with a name and value.

Since the relational data was verified, the resulting XML data should also be valid, assuming the correctness of the LINQ generation queries. However, comprehensive LINQ queries were also written to check the validity of the resulting heterogeneous data. The use of keys and referential integrity checking supported by XML technologies was explored for the XML documents but was not used due to the wide variability in support between the implementations of the specification. Fig. 6 summarizes the process used for generating the heterogeneous data sources. Once the generated data was bulk loaded into a relational database, the data was validated using LINQ queries and then XML data was generated using LINQ and subsequently validated. This data generation approach provides the ability to validate 


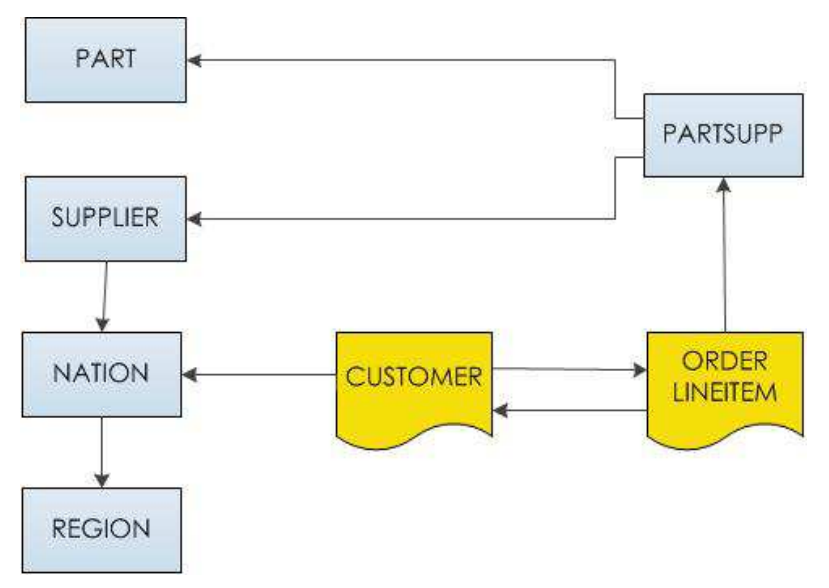

Figure 4: Diagram illustrating relational and XML data sources.

the results of the hybrid queries with the results of the TPC-H SQL queries against the relational database.

\section{LINQ Hybrid Query Design}

An analysis of the 22 decision support queries of the relational TPC-H benchmark illustrated the need for reformulation of the queries for the hybrid environment. This section details the restrictions and guidelines used for the hybrid query redesign.

LINQ data providers for hybrid queries over relational and XML data sources require that the first data source accessed be an XML data source. The data provider then coordinates the generation and evaluation of the underlying subqueries to the various sources. When a relational data source is listed first, the data providers assume that the query is only over a single relational database and an exception is generated. Thus, the benchmark queries were revised to reflect this requirement. In addition, there were several design decisions that arose during the query reformulation.

One goal of the design was to have the queries be independent (to the extent possible) of the particular product used to implement the relational database. The TPC-H scripts formed the basis of the relational component of the hybrid benchmark. The provided scripts used uppercase identifiers to define the attributes. Some databases respect the case of the attributes as given. Other databases can be configured for case sensitivity by a database 
new XElement ("CUSTOMERS"

from $c$ in CUSTOMER

select new XElement ("CUSTOMER",

new XElement ("C_CUSTKEY", c.C_CUSTKEY. ToString (). Trim ()),

... / / CUSTOMER attributes

new XElement("ORDERS",

from $O$ in ORDERS

where O.O_CUSTKEY $==$ c.C_CUSTKEY

select new XElement("O_ORDERKEY", o.O_ORDERKEY. ToString ( ) Trim ( )) )) ) ;

new XElement("ORDERS",

from $O$ in ORDERS

select new XElement("ORDER",

new XElement ("O_ORDERKEY", o.O_ORDERKEY.ToString ( ). Trim ( )), ... / / ORDERS attributes

new XElement("LINEITEMS"

from I in LINEITEM

where I.L_ORDERKEY == o. O_ORDERKEY

select new XElement("LINEITEM",

new XElement ("L_PARTKEY", I.L_PARTKEY. ToString

() . Trim ( ) ),

... // LINEITEM attributes

Figure 5: LINQ queries to generate XML data sources

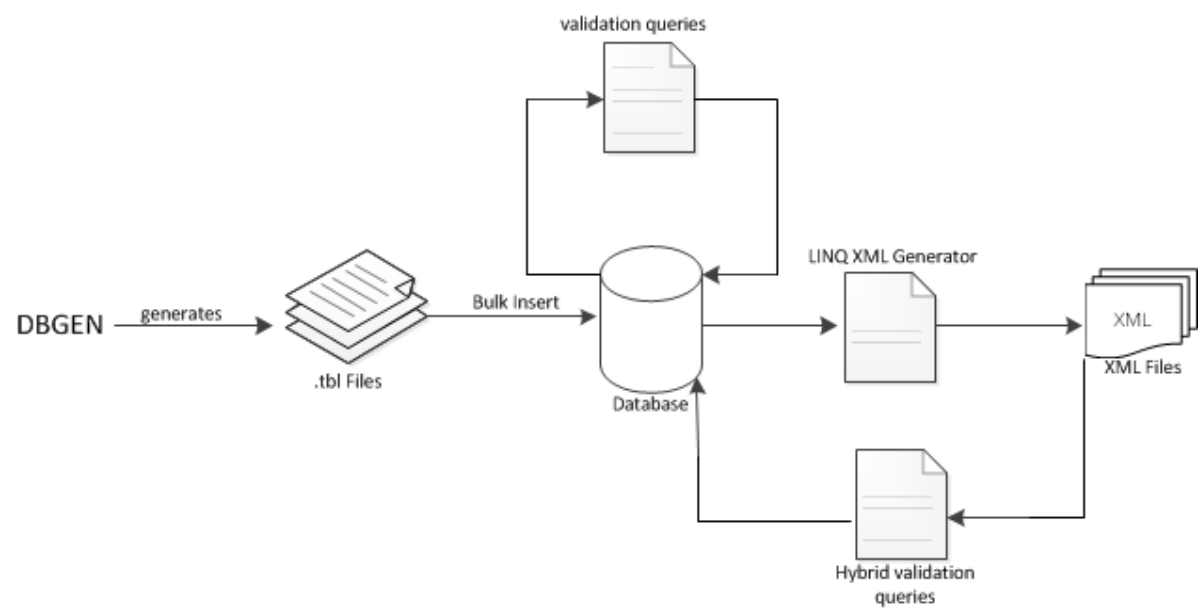

Figure 6: Overview of Process to Create and Validate Heterogeneous Data Sources. 


\begin{tabular}{|c|c|}
\hline TPC-H Query 5 & LINQ Hybrid \\
\hline 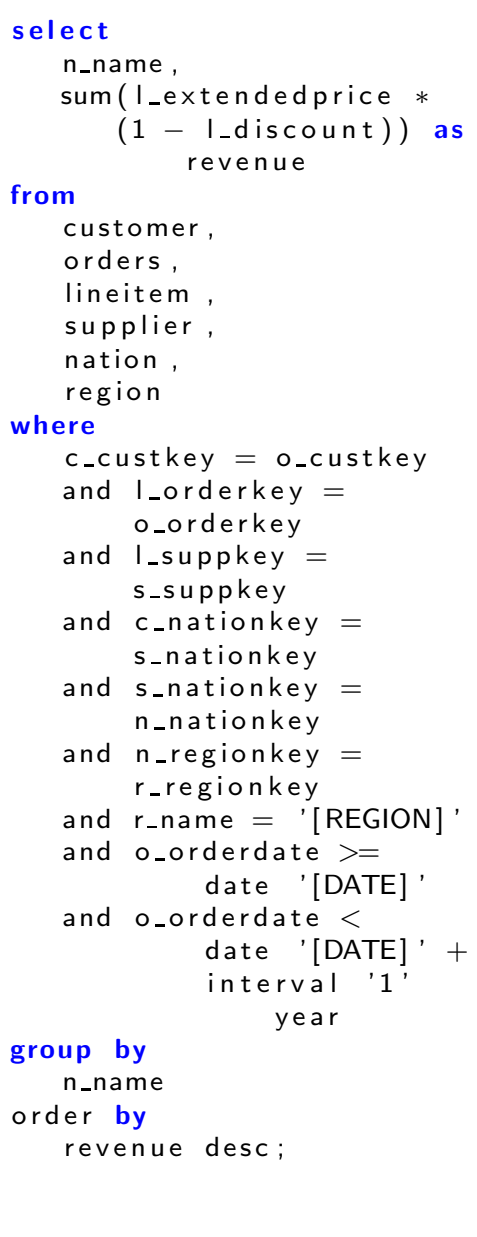 & 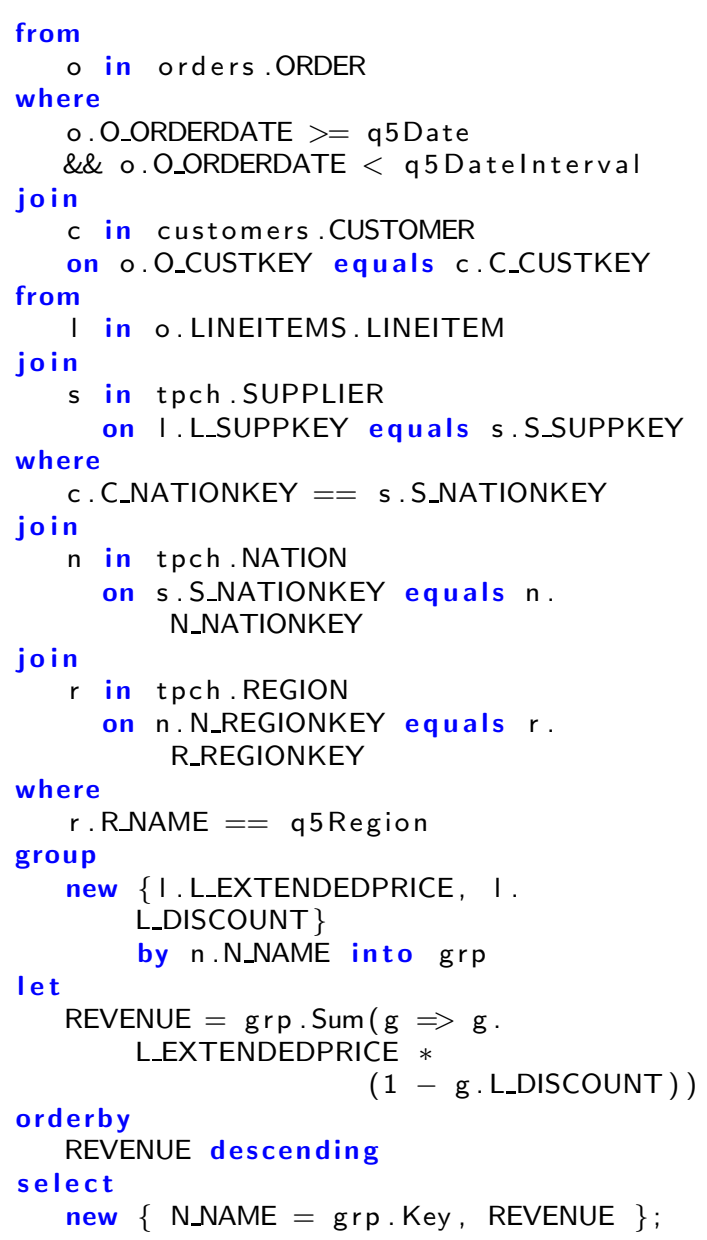 \\
\hline
\end{tabular}

Figure 7: TPC-H Query 5 Local Supplier Volume 
administrator. To allow for the use of the benchmark queries across database products and configurations, the hybrid queries use uppercase for the properties of the query result.

Query optimization is an important aspect of a query specification. In a purely relational environment such as LINQ to SQL, there is no apparent performance difference between using a join-on clause or a from-where clause to join two tables. This similarity is due to the query optimization performed by the underlying relational database, which chooses a join query plan in either of these cases. When using Entity Framework as the environment for executing the hybrid queries, there is a substantial performance difference between join-on versus from-where clauses. The join-on clause creates indexes to facilitate the join; the from-where clause does not create an index, so that the resulting evaluation uses a nested loop join approach, which is typically less efficient. Also, since there can be multiple where clauses intertwined between clauses of a LINQ query, the decision was made to move the where clauses as close as possible to the source that it filters. This process is analogous to pushing selections down a relational algebra query tree.

Another design decision involved grouping. Recall that LINQ provides the flexibility to specify what to group and what to group by. A design decision was made to only group information that was needed for the rest of the query. The grouping was essentially given by the original relational query specification in the benchmark specification [24]. For example, Fig. 7 shows the TPC-H Local Supplier Volume (Query 5) in both the SQL relational benchmark specification and the LINQ hybrid redesign, following the above restrictions and guidelines.

\section{Performance Evaluation}

The experimental methodology uses the design of the relational database and XML data described in Section 4 for a database instance that corresponds to the 200MB relational TPC-H. Fig. 8 shows more detailed information regarding the sizes of the tables and XML data for the evaluation. The performance measurements represent elapsed time for running the queries only for two database products. Fig. 9 provides a pictorial representation of the components of the experiment. Each database product resided on its own server, which consisted of a Dell PowerEdge R300 with a Dual core Xeon 3.0 GHz processor, 8 GB RAM, 2160 GB 7200 RPM Hard Drives and Dual Gigabit Network. The rest of the experimental environment resided 
on another server of the same characteristics. A C\# application provided the implementation of the LINQ queries as part of a project defined with the .NET Framework 4.0 in Visual Studio 2010 using the Entity Framework. The relational database accesses used LINQ to Entities with respective LINQ providers for the databases. Measurements were taken for both untyped XML using the LINQ to XML provider and typed XML using the LINQ to XSD provider. In addition, measurements were taken with and without clearing the database cache. The queries and data for running the benchmark are available on the Web [6].

\begin{tabular}{|l|r|r|}
\hline & \multicolumn{2}{|c|}{$200 \mathrm{MB}$} \\
\hline Data Source & $\#$ & Size (MB) \\
\hline \hline supplier & 2,000 & .320 \\
\hline part & 40,000 & 5.781 \\
\hline partsupp & 160,000 & 24.750 \\
\hline customer & 30,000 & 20.045 \\
\hline $\begin{array}{l}\text { orders } \\
\text { lineitems }\end{array}$ & 300,000 & 729.222 \\
\hline TOTAL SIZE & $1,199,969$ & \\
\hline
\end{tabular}

Figure 8: Data Source Sizes

The remainder of the section reports the performance measurements for the queries categorized by relational only queries, XML only queries, and hybrid queries. Note that all measurements represent the average of 20 runs of the query for that scenario. The parameter values used for the performance evaluation are the substitution values given in the original TPC-H specification for query validation of the $1 \mathrm{~GB}$ database instance. Due to the variability of the results, the scale of the y-axis was chosen for readability. Note that when the performance result extends beyond the represented yaxis maximum for the query set, the number of milliseconds to complete the query is indicated in text at the top of the bar for that query. In addition, an asterisk is used to indicate the few anomalous situations in which a query did not complete in a reasonable amount of time.

Fig. 10 provides a summary of characteristics for the benchmark queries to support the later discussion of the performance results. There are three types of joins that may occur in the queries: Relational, XML, and Hybrid. The relational join indicates that there exists at least one join between relational sources. The XML join represents a join between customers and orders, which 


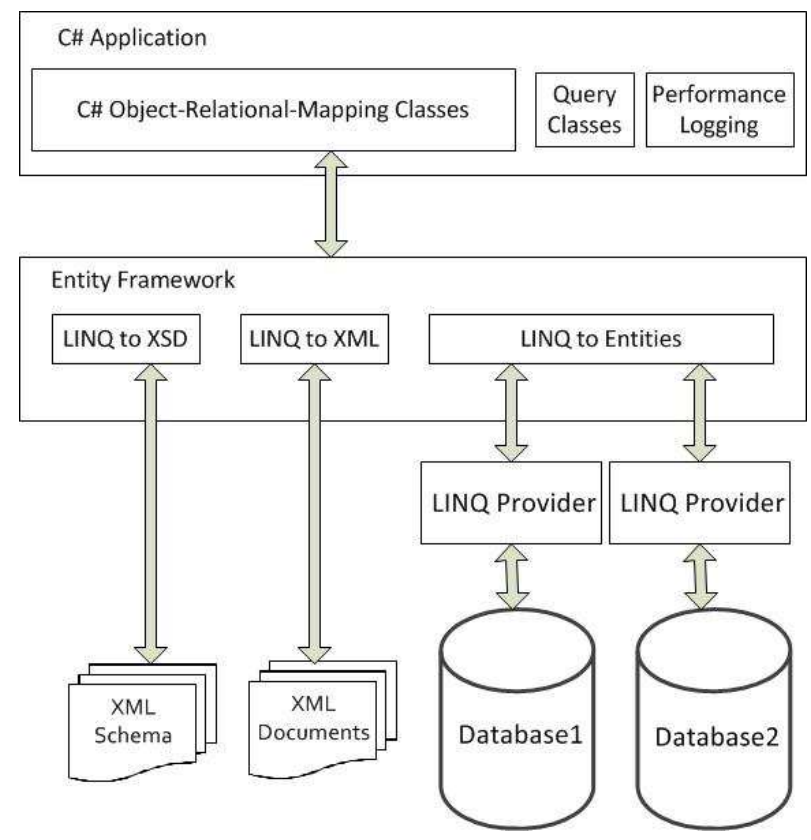

Figure 9: Hybrid Benchmark Stack.

are in separate XML documents. The hybrid join represents at least one join between XML and relational sources. There are four queries (Q1, Q4, Q6, Q12) that do not have any joins. These are XML only queries that use only the navigation between orders and the lineitems children. The aggregation column lists the aggregation operators used in the query. Grouping in LINQ specifies what to group and what to group by. The terms simple and complex are used to represent whether an existing field or identifier is used (simple) or a new anonymous object is created (complex). The comments column represents additional information, such as the use of helper queries or the Contains extension method.

\subsection{Relational Only Queries}

The queries from the hybrid version of the TPC-H benchmark queries that are relational only, include:

- Minimum Cost Supplier Query (Q2)

- Important Stock Identification Query (Q11) 


\begin{tabular}{|c|c|c|c|c|c|c|c|}
\hline & \multicolumn{3}{|c|}{ Join } & & \multicolumn{2}{c|}{ Grouping } & \\
\hline Query & Relational & XML & Hybrid & Aggregation & What & By & Comments \\
\hline \hline Q1 & & & & Average, Count, Sum & complex & complex & \\
\hline Q2 & $\checkmark$ & & & Min & & & \\
\hline Q3 & & $\checkmark$ & & Sum & simple & complex & \\
\hline Q4 & & & & Count & complex & simple & \\
\hline Q5 & $\checkmark$ & $\checkmark$ & $\checkmark$ & Sum & simple & simple & \\
\hline Q6 & & & & Sum & & & \\
\hline Q7 & $\checkmark$ & $\checkmark$ & $\checkmark$ & Sum & simple & complex & \\
\hline Q8 & $\checkmark$ & $\checkmark$ & $\checkmark$ & Sum & complex & simple & \\
\hline Q9 & $\checkmark$ & & $\checkmark$ & Sum & simple & complex & \\
\hline Q10 & & $\checkmark$ & $\checkmark$ & Sum & complex & complex & \\
\hline Q11 & $\checkmark$ & & & Sum & simple & simple & \\
\hline Q12 & & & & Count & complex & simple & \\
\hline Q13 & & $\checkmark$ & & Count & simple & simple & \\
\hline Q14 & & & $\checkmark$ & Sum & & & helper \\
\hline Q15 & & & $\checkmark$ & Max,Sum & complex & simple & helper \\
\hline Q16 & $\checkmark$ & & & Count, Distinct & simple & complex & Contains \\
\hline Q17 & & & $\checkmark$ & Average, Sum & & & \\
\hline Q18 & & $\checkmark$ & & Sum & simple & complex & \\
\hline Q19 & & & $\checkmark$ & Sum & & & \\
\hline Q20 & $\checkmark$ & & $\checkmark$ & Sum & & & Contains \\
\hline Q21 & $\checkmark$ & & $\checkmark$ & & simple & simple & \\
\hline Q22 & & $\checkmark$ & & Average & simple & simple & \\
\hline
\end{tabular}

Figure 10: Characteristics of Benchmark Queries 
- Parts/Supplier Relationship Query (Q16)

Fig. 11 charts the performance numbers for these queries over two database products for the largest $200 \mathrm{MB}$ database size for the four experimental possibilities, resulting from untyped versus typed XML and cache clearing versus no cache clearing. Note that although these are relational only queries, the measurements were taken for projects set up with these four alternatives and are reported consistently across all query categories. As expected the measurement of the options for untyped versus typed XML, which is not accessed in this category of queries, are comparable. The effect of cache clearing is evident in the performance measurements. In fact, Q16 in the second database product, labeled DB 2, did not complete in a reasonable amount of time within this environment when the cache was cleared, which is indicated by an asterisk in the chart.

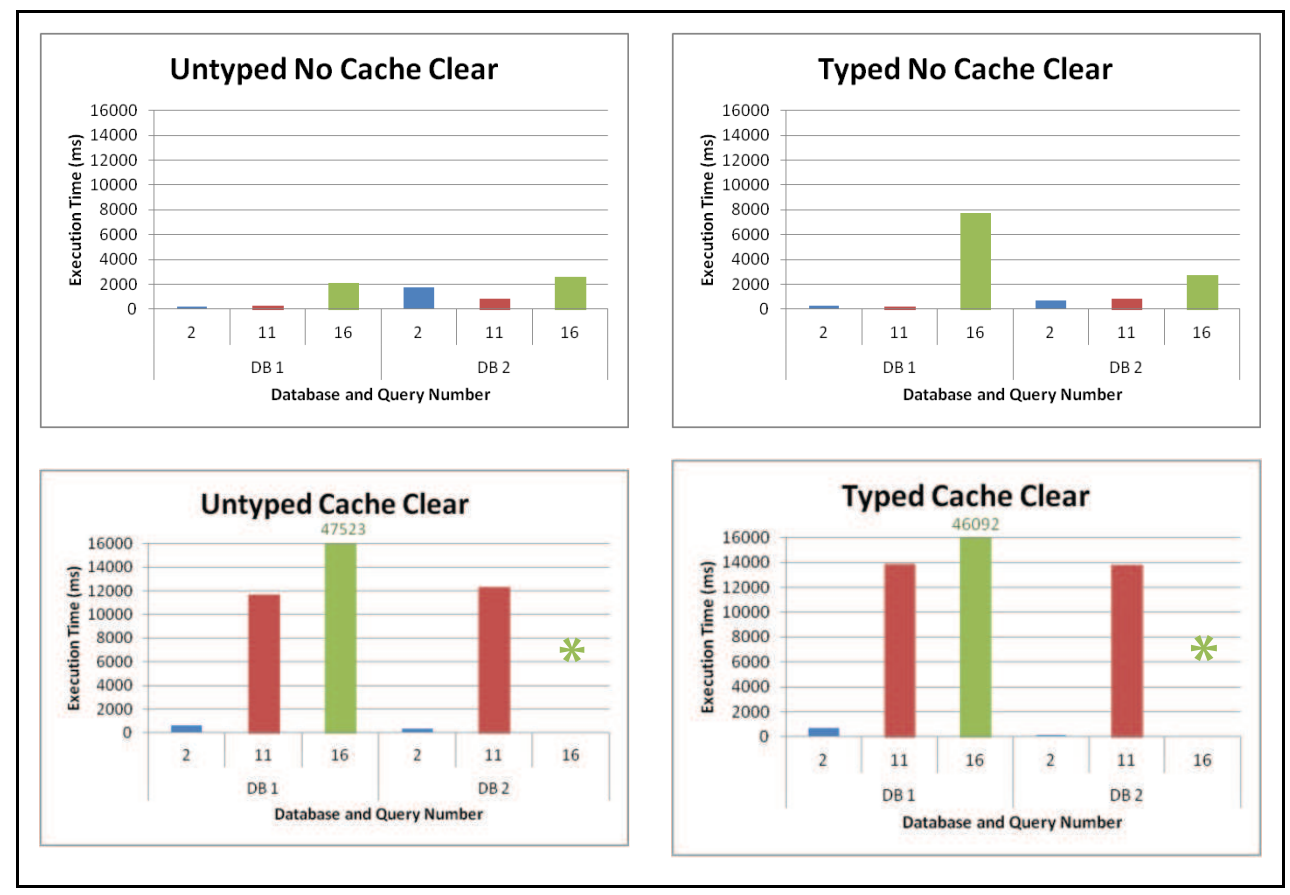

Figure 11: Relational Only Queries.

\subsection{XML Only Queries}

The hybrid version of the TPC-H benchmark queries include queries that access only XML sources: 
- Pricing Summary Report Query (Q1)

- Shipping Priority Query (Q3)

- Order Priority Checking Query (Q4)

- Forecasting Revenue Change Query (Q6)

- Shipping Modes and Order Priority Query (Q12)

- Customer Distribution Query (Q13)

- Large Volume Customer Query (Q18)

- Global Sales Opportunity Query (Q22)

Fig. 12 charts the performance numbers for these XML only queries. An examination of the four scenarios leads to some general conclusions. As expected, the relational cache clearing options had little effect on the XML only queries. For some of the queries (Q1, Q13, Q18, Q22) there appears to be a cost for using the typed version of the XML over both databases. Queries Q13, Q18, and Q22 involve an XML join, which may explain the overhead. However, Q3, which also uses an XML join, does not exhibit the performance penalty. Q1 also shows an increase in cost for the typed XML, although not as substantial as the other 3 queries. As shown in Fig. 10, Q1 uses three aggregation operators (Average, Count, Sum) and groups a new anonymous object by a new anonymous object.

\subsection{Hybrid Queries}

The queries that access both relational and XML sources from the hybrid version of the TPC-H benchmark queries include:

- Local Supplier Volume Query (Q5)

- Volume Shipping Query (Q7)

- National Market Share Query (Q8)

- Product Type Profit Measure Query (Q9)

- Returned Item Reporting Query (Q10)

- Promotion Effect Query (Q14) 


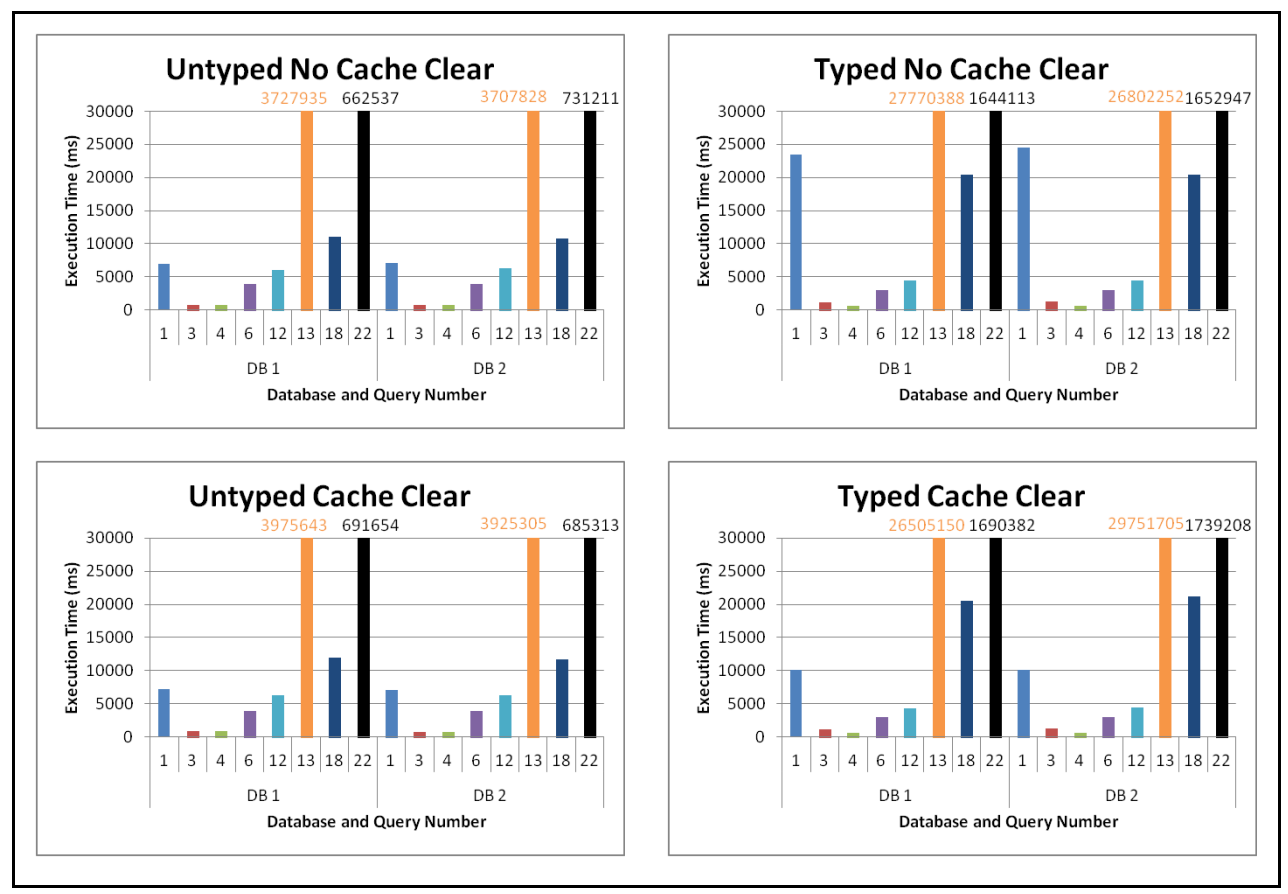

Figure 12: XML Only Queries. 


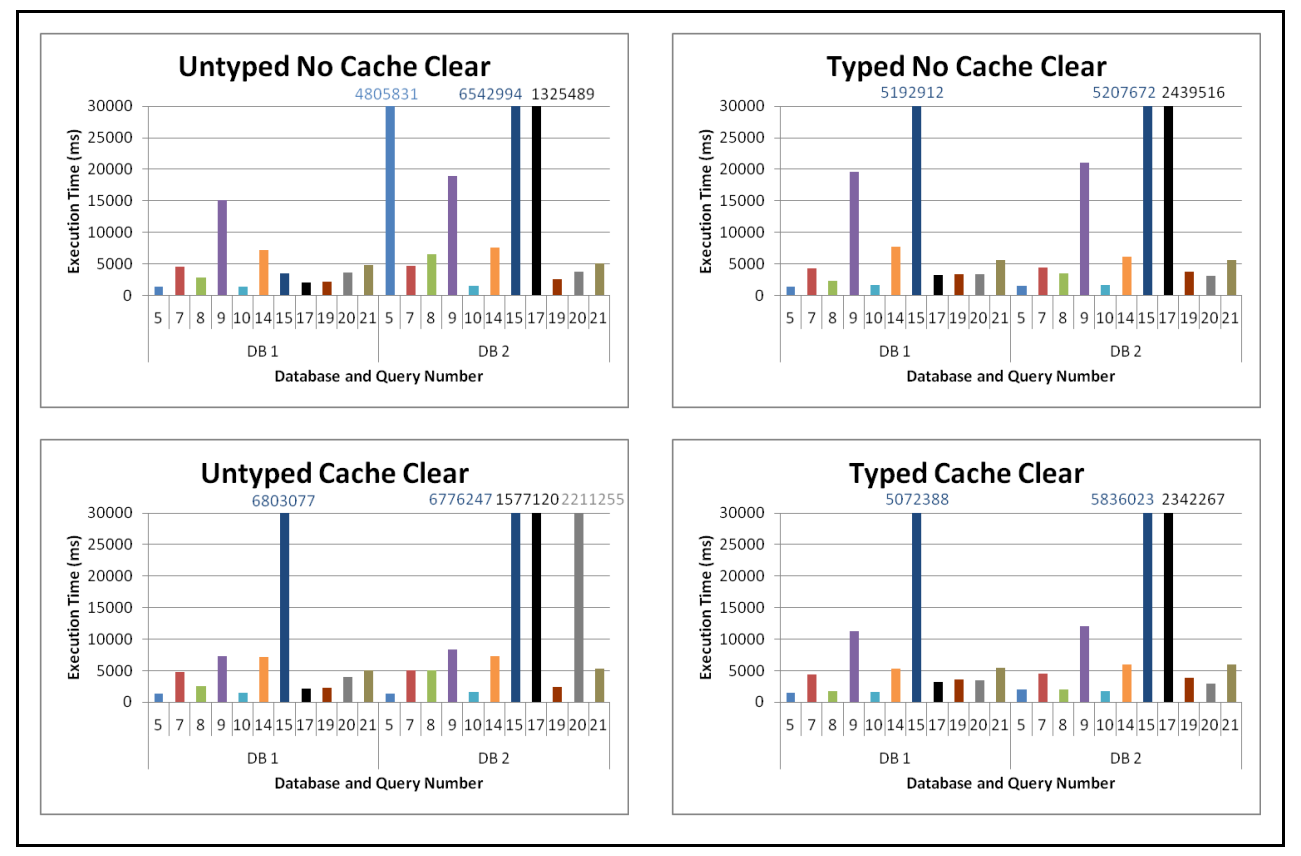

Figure 13: Hybrid Queries.

- Top Supplier Query (Q15)

- Small-Quantity-Order Revenue Query (Q17)

- Discounted Revenue Query (Q19)

- Potential Part Promotion Query (Q20)

- Suppliers Who Kept Orders Waiting Query (Q21)

Fig. 13 shows the performance results for the four scenarios over the 11 hybrid queries. Note that by definition all of the queries involve at least a join between a relational and XML source. There appears to be more variability in the performance for these queries including across database products. For both databases, Q9 exhibits overhead for the typed XML yet a performance improvement when the relational cache is cleared. Q15 requires a significant amount of time in all cases except for the untyped no cache clear scenario for DB 1. Similarly, Q5 is performant in all scenarios except for the untyped no cache clear scenario for DB 2. Q20 also exhibits a performance penalty in DB 2 for the untyped cache clear scenario. Looking at the summary 
characteristics table in Fig. 10, there does not appear to be any correlations to explain the anomalous results in general.

\section{Conclusions \& Future Work}

This paper presented the design of a hybrid relational and XML benchmark for loosely-coupled distributed data sources based on the TPC-H decision support benchmark. The data sources were designed such that the Web-based customer and order information was stored in XML documents and the remainder of the data were kept in relational tables. The 22 queries were redesigned in LINQ with the heterogeneous data sources, resulting in three relational only queries, eight XML only queries, and eleven hybrid queries that join relational and XML sources. Performance measurements for the query set were reported for two database products using untyped and typed XML with and without cache clearing. As expected, the caching on the relational only queries was quite effective. Some of the XML queries had a cost associated with using typed XML over untyped XML on both database products. The hybrid queries exhibited more variability in their performance even across the database products, which is probably due to the underlying data providers. Several versions of data providers for the database products were explored in an initial functional evaluation that tested the correctness of the query results using the original relational version of the TPC-H benchmark as a rubric.

This research is expected to provide contributions to the development of database performance benchmarks over heterogeneous data sources. A unique aspect of this work is its emphasis on combining both relational and XML data sources within the same benchmark and designing LINQ queries that access this heterogeneous data within a single query. There are other contributions from this research in terms of both testing and education. Since the TPC-H benchmark is a comprehensive decision support benchmark with complex queries involving aggregation and grouping, the resulting queries provide an environment in which to test both the functionality and efficiency of the LINQ providers for the database products. In addition, the hybrid benchmark data scenario was used as the basis for assessing the authors' research on materialized views over heterogeneous structured data sources in a distributed event stream processing environment $[4,3]$ that uses a data co-existence approach. The framework is based on distributed event processing agents that handle given responsibilities, leading towards common 
subexpressions across the distributed sources that can be materialized to improve performance [5]. Additionally, this research has also resulted in the incorporation of TPC-H and LINQ within the database curriculum [21, 7].

There are future research directions that can be pursued with respect to this initial benchmark that evaluates systems processing relational and XML data. The size of the relational data for the TPC-H benchmark used to populate the hybrid benchmark was $200 \mathrm{MB}$, which resulted in about $1 \mathrm{~GB}$ of data to process for the corresponding hybrid population. This restriction was necessary due to the amount of time it took to execute some of the queries. Therefore, one of the future research endeavors is to evaluate the benchmark with larger data sizes. One approach can investigate the use of Streaming classes that will stream the data to the queries [17]. Another possible research approach is to use the Ferry LINQ provider by [12], which avoids round trips to the data stores. In order to get better execution timings for the queries, one can also explore parallel processing using architectures such as PLinq [18].

\section{Acknowledgments}

This material is based upon work supported by the National Science Foundation under Grant No. CSR-0915325. Any opinions, findings, and conclusions or recommendations expressed in this material are those of the author(s) and do not necessarily reflect the views of the National Science Foundation.

\section{References}

[1] T. Böhme, E. Rahm, XMach-1: A benchmark for XML data management. In Andreas Heuer, Frank Leymann, and Denny Priebe, editors, BTW, Informatik Aktuell, pages 264-273. Springer, 2001.

[2] C. Calvert, D. Kulkarni, Essential LINQ. Addison-Wesley Professional, 2009.

[3] Chaudhari, M.B.: Materialized views over heterogeneous structured data sources in a distributed event stream processing environment. Ph.D. thesis, Arizona State University, Tempe, AZ, USA (2011) 
[4] M. B. Chaudhari, S. W. Dietrich, Metadata services for distributed event stream processing agents. In 19th International Conference on Software Engineering and Data Engineering, pages 307-312, San Francisco, 16-18 June 2010.

[5] M. B. Chaudhari, S. W. Dietrich, Detecting common subexpressions for multiple query optimization over loosely-coupled heterogeneous data sources. To appear in Distributed and Parallel Databases. http://dx.doi.org/10.1007/s10619-014-7166-6

[6] M. B. Chaudhari, S. W. Dietrich, J. Ortiz, Data and Queries for a Hybrid TPCH Benchmark over Heterogeneous Data Sources. Online: http://hdl.handle.net/2286/R.I.25515

[7] S. W. Dietrich and M. Chaudhari, LINQ ROX!: Integrating LINQ into the database curriculum. In Proceedings of the 42nd ACM technical symposium on Computer science education, SIGCSE '11, pages 293-298, New York, NY, USA, 2011. ACM.

[8] S. W. Dietrich, M. Brown, E. Cortes-Rello, S. Wunderlin, A practitioner's introduction to database performance benchmarks and measurements. Comput. J., 35(4):322-331, 1992.

[9] Elsayed, I., Brezany, P., Tjoa, A.M.: Towards realization of dataspaces. In: DEXA '06: Proceedings of the 17th International Conference on Database and Expert Systems Applications, pp. 266-272. IEEE Computer Society, Washington, DC, USA (2006). http://dx.doi.org/10.1109/DEXA.2006.140

[10] Franklin, M., Halevy, A., Maier, D.: From databases to dataspaces: a new abstraction for information management. SIGMOD Rec. 34(4), 27-33 (2005). http://doi.acm.org/10.1145/1107499.1107502

[11] J. Gray. Benchmark Handbook for Database and Transaction Processing Systems. Morgan Kaufmann, 1991.

[12] T. Grust, J. Rittinger, T. Schreiber, Avalanche-safe LINQ compilation. PVLDB, 3(1):162-172, 2010. 
[13] Halevy, A., Franklin, M., Maier, D.: Principles of dataspace systems. In: PODS '06: Proceedings of the twentyfifth ACM SIGMOD-SIGACT-SIGART symposium on Principles of database systems, pp. 1-9. ACM, New York, NY, USA (2006). http://doi.acm.org/10.1145/1142351.1142352

[14] F. Marguerie, S. Eichert, J. Wooley, LINQ in Action. Manning, 2008.

[15] E. Meijer, The world according to LINQ. Commun. ACM, 54(10):45-51, 2011.

[16] Microsoft. LINQ (Language INtegrated Query), 2014. msdn.microsoft.com/en-us/library/vstudio/bb397926.aspx.

[17] Microsoft .NET Framework: XStreamingElement Class., 2014. msdn.microsoft.com/enus/library/system.xml.linq.xstreamingelement(v=vs.110).aspx.

[18] Microsoft .NET Framework: Parallel LINQ (PLINQ)., 2014. msdn.microsoft.com/en-us/library/dd460688(v=vs.110).aspx.

[19] National Institutes of Health. National Information Exchange Model, 2015. http://www.niem.gov

[20] M. Nicola, I. Kogan, B. Schiefer, An XML transaction processing benchmark. In Chee Yong Chan, Beng Chin Ooi, and Aoying Zhou, editors, SIGMOD Conference, pages 937-948. ACM, 2007.

[21] J. Ortiz, S. W. Dietrich, M. B. Chaudhari, Learning from database performance benchmarks. J. Comput. Sci. Coll., 27(4):151-158, April 2012.

[22] A. Schmidt, F. Waas, M. L. Kersten, M. J. Carey, I. Manolescu, R. Busse, XMark: A benchmark for XML data management. In $V L D B$, pages 974-985. Morgan Kaufmann, 2002.

[23] Transaction processing performance council. TPC benchmark E: standard specification, revision 1.12.0, 2013. www.tpc.org/tpce.

[24] Transaction processing performance council. TPC benchmark H: standard specification, revision 2.16.0, 2013. www.tpc.org/tpch. 
[25] Xamarin. Mono, 2014. www.mono-project.com.

[26] XML database benchmark: Transaction processing over XML (TPoX), 2014. tpox.sourceforge.net.

\section{Vitae}

Dr. Mahesh B. Chaudhari is currently a sr. software engineer at Zephyr Health Inc. His primary responsibilities involve data modeling and enterprise integration, ontology development, algorithm design and incorporation into Zephyr Platform. Before Zephyr, he was a postdoctoral fellow at Arizona State University. He also has a Ph.D. in computer science from Arizona State University. His academic research interests focus on incremental view maintenance, condition monitoring over distributed heterogeneous data sources, and multiple query optimization. He has 2 years of teaching experience and is a recipient of PFF Emeriti Fellowship for excellence in research, teaching and mentorship.

Dr. Suzanne W. Dietrich is a Professor in the Applied Computing program within the School of Mathematical and Natural Sciences at Arizona State University. She has an established research foundation covering materialized view maintenance in various environments including active rules and event processing, with an emphasis in performance evaluation. Her research has also included event-based application integration using rules.

Jennifer Ortiz is currently a Computer Science PhD student at the University of Washington. She is researching the development of tools to facilitate analysis of scientific data in the cloud. Jennifer has an MS in Computer Science from the University of Washington. Jennifer contributed to this paper as an undergraduate student in Applied Computing at Arizona State University.

Spencer Pearson contributed to this paper as an undergraduate student at Arizona State University. His research interests include Similarity Joins and other similarity-aware operators. He graduated from Arizona State University with a BS in Applied Computing as part of Barrett, the Honors College. 